\title{
Co-Delivery of Curcumin and Cisplatin to Enhance Cytotoxicity of Cisplatin Using Lipid-Chitosan Hybrid Nanoparticles
}

This article was published in the following Dove Press journal: International Journal of Nanomedicine

\author{
Muhammad Muzamil Khan' \\ Asadullah Madni (1D \\ Nayab Tahir ${ }^{2}$ \\ Farzana Parveen' \\ Safiullah Khan' \\ Nasrullah Jan' \\ Ahsan $\mathrm{Ali}^{\mathrm{I}}$ \\ Muhammad Abdurrahim ' \\ Umar Farooq (iD ${ }^{3}$ \\ Muhammad Imran Khan (D) ${ }^{4}$ \\ 'Department of Pharmacy, The Islamia \\ University of Bahawalpur, Bahawalpur, \\ Pakistan; ${ }^{2}$ College of Pharmacy, \\ University of Sargodha, Sargodha, \\ Pakistan; ${ }^{3}$ Department of Pharmacy, \\ Government College University \\ Faisalabad, Faisalabad, Pakistan; ${ }^{4}$ Faculty \\ of Pharmacy, Riphah International \\ University, Lahore, Pakistan
}

Background: Lipid-polymer hybrid nanoparticles (LPHNP) are suitable for co-delivery of hydrophilic and lipophilic drugs. The structural advantages of polymers and biomimetic properties of lipids enable higher encapsulation of drugs and controlled release profile. Lipidpolymer hybrid nanoparticles have been prepared for co-delivery of curcumin and cisplatin for enhanced cytotoxicity against ovarian cancer.

Material and Methods: Chitosan, cisplatin, curcumin, Lipoid S75 were selected as structural components and ionic gelation method was used for preparation of LPHNPs. Nanoparticles were formed via ionic interaction of positively charged chitosan and negatively charged lipid.

Results: The optimized nanoparticles were of $225 \mathrm{~nm}$ with cationic charge. The encapsulation efficiency was greater than $80 \%$ with good drug loading. The drug release profile showed controlled release behavior of both curcumin and cisplatin simultaneously and the absence of burst release. The in vitro therapeutic efficacy and cellular association was evaluated using A2780 ovarian cell lines. To further investigate therapeutic efficacy, we developed 3D spheroids as tumor model to mimic the in vivo conditions. The cytotoxicity and uptake of co-loaded LPHNPs were evaluated on 3D spheroids and results indicated increased chemosensitization and enhanced therapeutic efficacy of co-loaded LPHNPs.

Conclusion: Lipid-polymer hybrid nanoparticles could be a suitable platform for co-delivery of curcumin and cisplatin for enhanced cytotoxic effect on ovarian cell lines.

Keywords: co-delivery, chemosensitization, curcumin, cisplatin, enhanced therapeutic output

\section{Introduction}

Cancer is one of the leading causes of death in the world and chemotherapy is frequently used treatment for the management of cancer. There are various chemotherapeutic agents approved for the treatment of cancer but they lack specificity and are generally less tolerable. ${ }^{1}$ Ovarian cancer is amongst the deadliest cancers in women and is one of the leading causes of death. ${ }^{2}$ Treatments include de-bulking surgery and platinum-based chemotherapy. ${ }^{3}$ Despite a successful initial response from platinumbased drugs, tumors develop resistance and lead to reduced response. ${ }^{4}$

To avoid these drawbacks of chemotherapy, it is necessary to incorporate natural therapeutic moieties having the potential to reduce tumor growth. ${ }^{5}$ It is of prime importance to explore the natural products having tumor inhibitory properties without harming healthy cells. ${ }^{6}$ Some natural products are also known to increase chemosensitization of the tumor and enhance the effectiveness of chemotherapeutic agents without harming normal body cells. ${ }^{7}$
Correspondence: Muhammad Muzam Khan

The Islamia University of Bahawalpur, Bahawalpur, Punjab 63100, Pakistan Email muzamilpharmacist@gmail.com

Asadullah Madni

Department of Pharmacy, The Islamia University of Bahawalpur, Bahawalpur

Punjab 63100, Pakistan

Tel +923366248108

Email asadpharmacist@hotmail.com 
Curcumin is a phenolic derivative of turmeric. Curcumin is used as a therapeutic agent in various diseases including cancer. Curcumin has antiproliferative effect on breast cancer cell lines by inhibiting the orthenine decarboxylase activity. ${ }^{8}$ Curcumin also has chemopreventive effect against breast cancer. ${ }^{9}$ Some in-vivo studies support the antimetastatic role of curcumin. ${ }^{10}$ Several studies also support the therapeutic role of curcumin in ovarian cancer. ${ }^{11,12}$ Curcumin increases the sensitivity of the standard chemotherapy regime in resistant cancer cells. The anti-proliferative effect of curcumin is enhanced in combination with chemotherapeutic agents. ${ }^{13}$ Curcumin inhibits the cell cycle at the $\mathrm{G}_{2} / \mathrm{M}$ phase and renders the cells more sensitive to radiation. Curcumin induces apoptosis in cancer cells by inhibiting the DNA topoisomerase II. ${ }^{14}$

The conventional administration of cisplatin in ovarian cancer produces severe side effects on vital organs such as kidney and liver. In recent era, various efforts have been made to reduce the undesirable effects by encapsulating in micelles, polymeric nanoparticles or coating with the lipids. ${ }^{15}$ Some studies support the role of curcumin to increase the chemosensitization of ovarian cancer and enhance the effectiveness of cisplatin on the ovarian cell lines. ${ }^{16}$ Curcumin is also known to reduce nephrotoxicity ${ }^{17}$ and ototoxicity caused by cisplatin. ${ }^{18}$ Curcumin overcomes the resistance of cisplatin and increases the therapeutic efficacy of cisplatin. ${ }^{19}$

The conventional drug delivery system results in a burst release of chemotherapeutic agents, which may further increase side effects. Nanotechnology enables the drug to be delivered at the targeted site in a controlled manner. ${ }^{20}$ Nanoparticle-based systems enable the delivery of therapeutic agents to the specific cancer cells resulting in increased therapeutic benefits. ${ }^{21}$

Lipid-polymer hybrid nanoparticles (LPHNPs) combine the structural advantage of polymer and biomimetic properties of lipids and enable the controlled release of drugs with high encapsulation efficiency. ${ }^{22}$ Lipid-polymer hybrid drug delivery system can incorporate hydrophilic and lipophilic drugs at the same time. ${ }^{23}$ Although polymeric nanoparticles and liposomes have been effectively used for drug delivery, but they suffer some drawbacks such as the stability problem of liposomes and aggregation of polymeric nanoparticles. To overcome these drawbacks associated with polymeric and liposomal drug delivery system we developed a lipid-polymer hybrid system for enhanced therapeutic outcomes. ${ }^{24}$
Chitosan is a suitable polymer for drug delivery due to its biodegradability and low cost. ${ }^{25}$ Chitosan is a biocompatible polymer obtained from chitin. It has anionic drug delivery properties because of its cationic charge and can form complexes with anionic drugs such as naproxen ${ }^{26}$ and enoxaparin. ${ }^{27}$ Chitosan is also a suitable polymer for controlled drug delivery. ${ }^{28}$ Here we use chitosan as a polymer and Lipoid S75 as a lipid to prepare lipid-polymer hybrid nanoparticles for dual delivery of cisplatin and curcumin to enhance chemosensitization of tumor cell lines and increase the therapeutic output of cisplatin.

\section{Materials and Methods Materials}

Curcumin, Chitosan (Low molecular weight: 50,000 190,000 Da), MCF-7 breast cell lines and A2780 ovarian cell lines were purchased from Sigma Aldrich (Chemie, Germany). LIPOID S75 was received from the LIPOID (Newark, NJ, USA). Cisplatin was received as a gift sample from Pharmedic Laboratories (Pvt) Ltd, Pakistan. Ethanol and acetic acid were of analytical grade and purchased from Fisher Scientific (MA, USA). Cell Titer Blue ${ }^{\circledR}$ and Cell Titer Glo ${ }^{\circledR}$ were purchased from Promega ${ }^{\circledR}$ (Wisconsin, USA). Roswell Park Memorial Institute Medium (RPMI), fetal bovine serum (FBS), and antimycotic, antibacterial solutions were obtained from CellGro (VA, USA). HOECHST 3342 was purchased from Molecular Probes Inc. (Eugene, OR). Paraformaldehyde was from Electron Microscopy Sciences (Hatfield, PA, USA). Trypan blue solution was obtained from Hyclone (Logan, UT, USA). Fluorescent dyes Rhodamine123 and Rhodamine-PE were purchased from Sigma Aldrich (MA. USA). Flow cytometry (Beckton Dickinson FACS Calibur TM, NJ, USA) was used and Plate reader (Biotek) was used for cell count.

\section{Preparation of Cisplatin and Curcumin Loaded LPHNPs}

LPHNPs were prepared by ionic gelation method as described by Sonvico et $\mathrm{al}^{29}$ Briefly, $2.5 \mathrm{mg}$ of chitosan was dissolved in $23 \mathrm{~mL}$ of acetic acid solution with an overnight stirring and cisplatin was dissolved in the same solution while $50 \mathrm{mg}$ of lipid was dissolved in $2 \mathrm{~mL}$ of ethanol and curcumin was then dissolved in the same ethanolic solution. The ethanolic solution was added dropwise into acetic acid solution with constant stirring at 1000rpm for 30 mins. The lipid-polymer hybrid nanoparticles (LPHNPs) were formulated by the interaction of 
positively charged chitosan and negatively charged lipids (Figure 1). The LPHNPs with varying ratios of lipid to chitosan were prepared and were then centrifuged at 10,000rpm for 30 mins for further characterization.

The fluorescent dyes ( 0.5 mole percent) along with lipids were also dissolved in ethanolic solution to prepare nanoparticles for cell uptake studies. For cisplatin loaded LPHNPs only cisplatin was dissolved in acetic acid solution and for curcumin loaded LPHNPs only curcumin was dissolved in an ethanolic solution of lipid.

\section{Size and Zeta Potential}

The prepared LPHNPs were analyzed for size and surface charge using Zeta Sizer (Malvern, UK). The dynamic light scattering technique was used for the determination of size. $50 \mu \mathrm{L}$ of nanoparticle solution was placed in a cuvette and size was determined. Phosphate buffer saline was used for dilution to measure the surface charge. Measurement was performed at room temperature at $90^{\circ}$ scattering angle and three readings were taken for each sample.

\section{Entrapment Efficiency and Drug Loading}

The entrapment of cisplatin and curcumin inside the lipid polymer hybrid nanoparticles was determined by an indirect method. ${ }^{30}$ The LPHNPs were prepared and centrifuged at $10,000 \mathrm{rpm}$, supernatant was used to measure the amount of un-entrapped drugs in the nanoparticles. The amount of un-entrapped drugs was measured using a UV spectrophotometer at $210 \mathrm{~nm}$ for cisplatin ${ }^{31}$ and $420 \mathrm{~nm}$ for curcumin. ${ }^{32}$ The entrapment efficiency and drug loading of cisplatin and curcumin was determined by following formulas:

$$
\begin{aligned}
\text { Entrapment efficiency }(\%)= & \text { amount of entrapped drug/Theoretical } \\
& \text { amount of drug } \times 100
\end{aligned}
$$

Drug loading (\%) = Mass of drug in nanoparticles/mass of nanoparticles $\times 100$

\section{Transmission Electron Microscopy}

The morphology of prepared LPHNPs was analyzed by using the transmission electron microscope (Jeol, USA). The samples of cisplatin and curcumin co-loaded LPHNPs were applied directly on the grid and suitable images were taken at different resolutions.

\section{In vitro Drug Release}

The drug release studies were performed at $\mathrm{pH} 7.4$ using the dialysis bag method. The dialysis membrane with MWCO 10-12 kDa was used. The prepared hybrid nanoparticles loaded with $5 \mathrm{mg}$ of cisplatin in chitosan solution and $5 \mathrm{mg}$ of curcumin in lipid solution were suspended inside the dialysis bag and immersed in the phosphate buffer saline at $37^{\circ} \mathrm{C}$ and $100 \mathrm{rpm}$. The samples were withdrawn at predetermined time intervals and the contents of cisplatin and curcumin were analyzed using the UV spectrophotometer.

\section{Cell Cytotoxicity}

Cell viability studies were performed on ovarian cell lines to evaluate the therapeutic efficacy of hybrid nanoparticles. A2780 cell lines were used. Cells (5000) were seeded in each well of a 96 well plate and incubated at $25^{\circ} \mathrm{C}$ in $5 \% \mathrm{CO}_{2}$. After overnight incubation the cells were treated with curcumin loaded LPHNPs, cisplatin loaded LPHNPs and co-loaded (Cisplatin+ curcumin) LPHNPs to observe

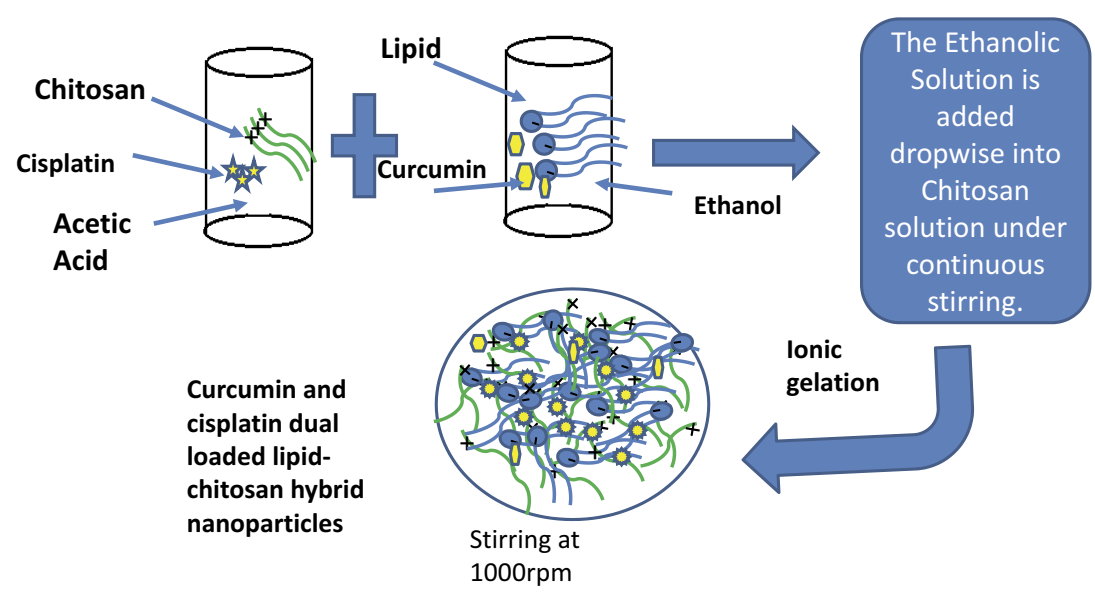

Figure I Schematic diagram of formation of curcumin and cisplatin co-loaded LPHNPs. 
the increased therapeutic effect of curcumin. After $4 \mathrm{hrs}$ of treatment, the formulations were removed and replaced with fresh media and cell viability was observed using Cell Titer Blue counted by the plate reader (BioTek) after $24 \mathrm{hrs}$ and $48 \mathrm{hrs}$ of treatment. ${ }^{33}$

\section{Cell Uptake}

Cell uptake studies were performed to measure the uptake of LPHNPs. Cells $(500,000)$ were seeded in each well of a 6 well plate. After $24 \mathrm{hrs}$ of incubation, cells were treated with co-loaded (cisplatin+curcumin) LPHNPs having rhodamine123 and cisplatin loaded LPHNPs with Rhodamine-123. After $4 \mathrm{hrs}$ of treatment, the cells were collected using trypsin and washed with PBS and then re-suspended in PBS and uptake of hybrid nanoparticles was measured using flow cytometry.

\section{Fluorescence Microscopy}

Cells $(200,000)$ were seeded in each well of a 12 well plate containing microscopic glass slide. After overnight incubation, the cells were treated with co-loaded LPHNPs to observe the qualitative uptake by the cells. The fluorescent dyes rhodamine-123 and rhodamine-PE were incorporated inside the co-loaded LPHNPs and fluorescence was observed. After $4 \mathrm{hrs}$ of treatment, the cells were collected and fixed using $4 \%$ paraformaldehyde and then treated with HOECHST 33342 and washed with PBS and mounted on microscopic slides using a mounting buffer and observed using KEYENCE (BZX710) fluorescence microscope.

\section{Preparation of 3D Spheroids}

The spheroids were prepared using MCF-7 breast cell lines. A 96 well plate was first coated with agarose $15 \%$ and cells (7000) were seeded in each well of a 96 well plate and centrifuged to create the collective layers of cells and placed in incubator. The plates were observed under the microscope for the development of spheroids and fresh media was replaced on alternative days for the growth of spheroids until the formation of $3 \mathrm{D}$ spheroids. ${ }^{34}$

\section{Cytotoxicity Studies Toward 3D Spheroids}

The prepared spheroids were used to evaluate the therapeutic efficacy of LPHNPs and to measure the effect of added curcumin to the cisplatin loaded LPHNPs. The spheroids were treated with cisplatin loaded LPHNPs, curcumin and cisplatin co-loaded LPHNPs and cisplatin solution. After 24 and 48 hrs of treatment, the spheroids were collected and treated with Cell Titer Glo (CTG) protocol as per manufacturer instructions and cytotoxicity was evaluated using a plate reader (BioTek).

\section{Cell Uptake Studies Using 3D Spheroids}

The uptake of hybrid nanoparticles by 3D spheroids was evaluated quantitatively using flow cytometry. The LPHNPs were loaded with fluorescent dye rhodamine-PE to measure the uptake. The spheroids were treated with curcumin and cisplatin co-loaded LPHNPs and cisplatin loaded LPHNPs and after $4 \mathrm{hrs}$ of treatment the cells were collected centrifuged and re-suspended in $300 \mu \mathrm{L}$ of PBS and fluorescence was measured using flow cytometry. ${ }^{34}$

\section{Fluorescence Imaging of 3D Spheroids}

The prepared 3D spheroids were treated with co-loaded LPHNPs to evaluate the qualitative uptake of LPHNPs by 3D spheroids. After $4 \mathrm{hrs}$ of treatment, the spheroids were collected and fixed with $4 \%$ paraformaldehyde and kept at $4^{\circ} \mathrm{C}$ overnight. The spheroids were then treated with HOECHST 33342 and suitable images were taken using the fluorescence microscopy.

\section{Results and Discussion}

\section{Average Particle Size and Zeta Potential}

The size and zeta potential of LPHNPs were determined by using the dynamic light scattering technique. The results indicated that the size of all the formulations with various ratios of lipid to chitosan was in the nanometer range and varies from 200 to $700 \mathrm{~nm}$. The surface charge of formulations remained positive till the 40:1 ratio of lipid to chitosan due to the cationic nature of chitosan and hybrid nanoparticles with lipid to chitosan ratio of 60:1 showed negative charge due to higher contents of lipids (Table 1). ${ }^{35}$ The positive charge on the nanoparticles progressively decreases with an increase in lipid concentration. At lipid to chitosan ratio of 20:1, the hybrid nanoparticles were of suitable size with lower PDI and cationic surface charge.

\section{Entrapment Efficiency and Drug Loading}

The entrapment efficiency of prepared LPHNPs was calculated by the indirect method. LPHNPs with different ratios of lipid to chitosan showed more than $75 \%$ encapsulation efficiency and nanoparticles with lipid to chitosan ratio of $20: 1$ showed more than $85 \%$ encapsulation 
Table I Size and Surface Charge of Cisplatin and Curcumin Dual Loaded LPHNPs. Results Indicate Mean $\pm S D, n=3$

\begin{tabular}{|l|l|l|l|}
\hline $\begin{array}{l}\text { Lipid:Polymer } \\
\text { Ratio }\end{array}$ & Size (nm) & PDI & $\begin{array}{l}\text { Potential } \\
(\mathbf{m V})\end{array}$ \\
\hline $5: 1$ & $437.3 \pm 2.2$ & $0.32 \pm 0.02$ & $45.5 \pm 1.8$ \\
$10: 1$ & $276.7 \pm 2.8$ & $0.29 \pm 0.03$ & $45.2 \pm 1.3$ \\
$20: 1$ & $225.5 \pm 0.8$ & $0.21 \pm 0.01$ & $32.6 \pm 0.7$ \\
$30: 1$ & $392.7 \pm 1.2$ & $0.33 \pm 0.04$ & $30.3 \pm 1.2$ \\
$40: 1$ & $353.9 \pm 1.4$ & $0.35 \pm 0.05$ & $21.6 \pm 1.1$ \\
$60: 1$ & $680.1 \pm 1.1$ & $0.19 \pm 0.07$ & $-0.21 \pm 0.6$ \\
\hline
\end{tabular}

Table 2 Encapsulation Efficiency and Drug Loading of Cisplatin and Curcumin. Results Indicate Mean $\pm S D, n=3$

\begin{tabular}{|l|l|l|l|l|}
\hline $\begin{array}{l}\text { Lipid: } \\
\text { Polymer } \\
\text { Ratio }\end{array}$ & $\begin{array}{l}\text { EE (\%) } \\
\text { Curcumin }\end{array}$ & $\begin{array}{l}\text { DL (\%) } \\
\text { Curcumin }\end{array}$ & $\begin{array}{l}\text { EE (\%) } \\
\text { Cisplatin }\end{array}$ & $\begin{array}{l}\text { DL (\%) } \\
\text { Cisplatin }\end{array}$ \\
\hline $5: 1$ & $60.3 \pm 2.2$ & $1.1 \pm 0.4$ & $79.5 \pm 2.3$ & $1.5 \pm 0.4$ \\
$10: 1$ & $67.7 \pm 2.8$ & $1.2 \pm 0.3$ & $81.2 \pm 1.8$ & $1.4 \pm 0.3$ \\
$20: 1$ & $78.5 \pm 0.8$ & $2.1 \pm 0.2$ & $85.6 \pm 0.9$ & $2.3 \pm 0.2$ \\
$30: 1$ & $75.7 \pm 1.2$ & $1.4 \pm 0.3$ & $82.1 \pm 1.5$ & $1.8 \pm 0.3$ \\
$40: 1$ & $77.9 \pm 1.4$ & $2.1 \pm 0.5$ & $76.5 \pm 1.1$ & $1.7 \pm 0.5$ \\
$60: 1$ & $81.1 \pm 1.1$ & $2.5 \pm 0.6$ & $75.1 \pm 1.4$ & $1.5 \pm 0.6$ \\
\hline
\end{tabular}

efficiency (Table 2). The encapsulation efficiency is higher than polymeric nanoparticles and is attributed to the presence of lipid layer. The formulations with a lower ratio of lipids showed lower encapsulation of curcumin, as the ratio of lipid is increased the encapsulation efficiency of curcumin is increased because of its better solubility in lipophilic phase and incorporation inside the hybrid system. The hybrid nanoparticles system serves as a reservoir and incorporate both lipophilic and hydrophilic drugs with high encapsulation efficiency and avoid irregular drug release. ${ }^{36}$ The hybrid nanoparticles with lipid to chitosan ratio of 20:1 were selected for further studies.

\section{Morphology}

The morphology of LPHNPs was evaluated using the transmission electron microscope (Jeol, USA). The images showed spherical-shaped nanoparticles (Figure 2). The size of LPHNPs was in the range of $225 \mathrm{~nm}$, which is in accordance with the size determined by dynamic light scattering technique. The morphology of curcumin and cisplatin coloaded nanoparticles was similar to previously reported with curcumin and doxorubicin co-loaded nanoparticles. ${ }^{37,38}$ The lipid and chitosan interact with each other via ionic gelation and form spherical-shaped nanoparticles.

\section{In vitro Drug Release Studies}

The release of both cisplatin and curcumin was observed using the dialysis bag method. Phosphate buffer saline $\mathrm{pH}$ 7.4 was used as a dissolution medium. ${ }^{39}$ There was not any burst release of cisplatin or curcumin from the LPHNPs. The results showed that both cisplatin and curcumin released in a controlled manner from lipid-chitosan hybrid nanoparticles while there is some burst release in the case of free cisplatin and curcumin. The results indicated that only $50 \%$ of curcumin is released from hybrid nanoparticles in $24 \mathrm{hrs}$ and controlled release behavior of curcumin from nanoparticles was observed, which is also reported in previous chitosan nanoparticles loaded with curcumin. ${ }^{40}$ The release of cisplatin was also controlled and only $68 \%$ of cisplatin in released in $24 \mathrm{hrs}$ (Figure 3). This is suitable for the sustained delivery of drugs and to avoid a higher therapeutic level. The lipid-chitosan hybrid nanoparticles are suitable for the controlled release of both

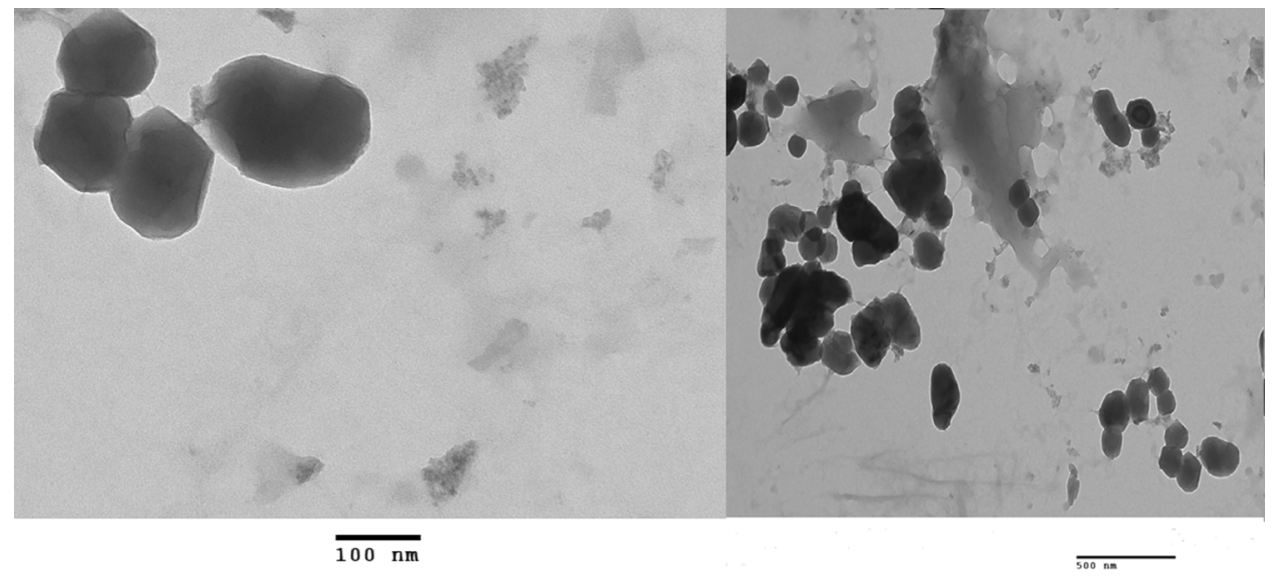

Figure 2 Transmission electron microscopy images of curcumin and cisplatin co-loaded LPHNPs. 

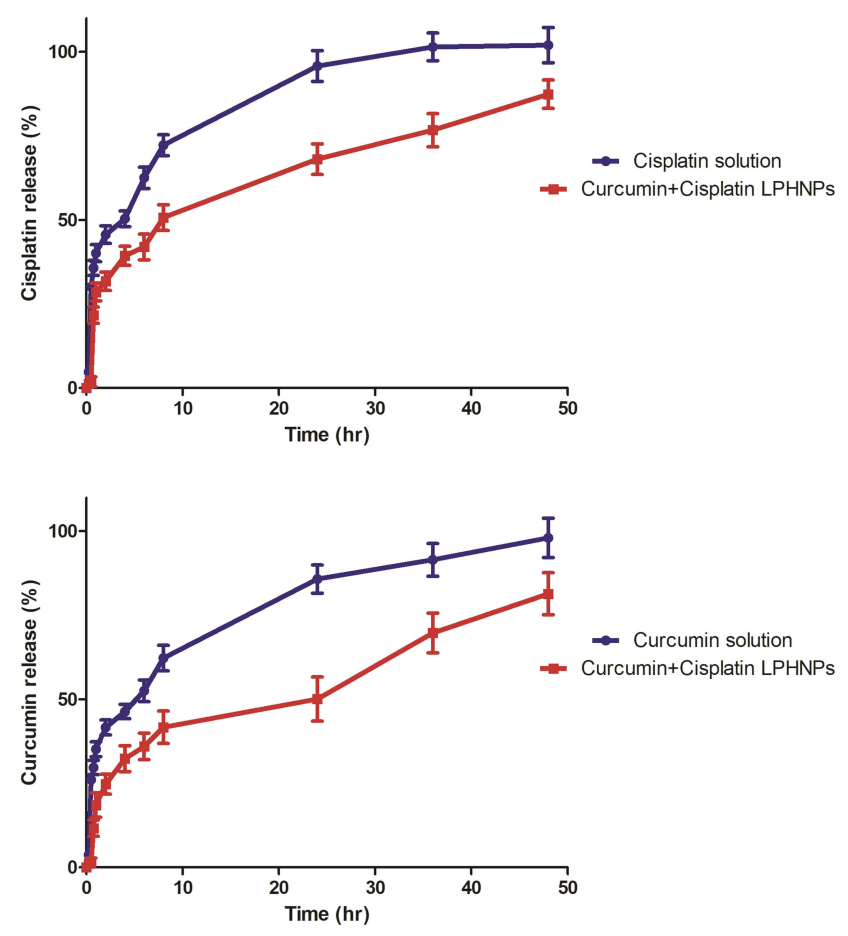

Figure 3 Drug release profile of cisplatin and curcumin from LPHNPs.

lipophilic and hydrophilic drugs. The polymer provides higher encapsulation and avoids burst release of drug and lipid layer provides a diffusional barrier that further enhances the controlled release behavior of drug from hybrid nanoparticles. ${ }^{41}$
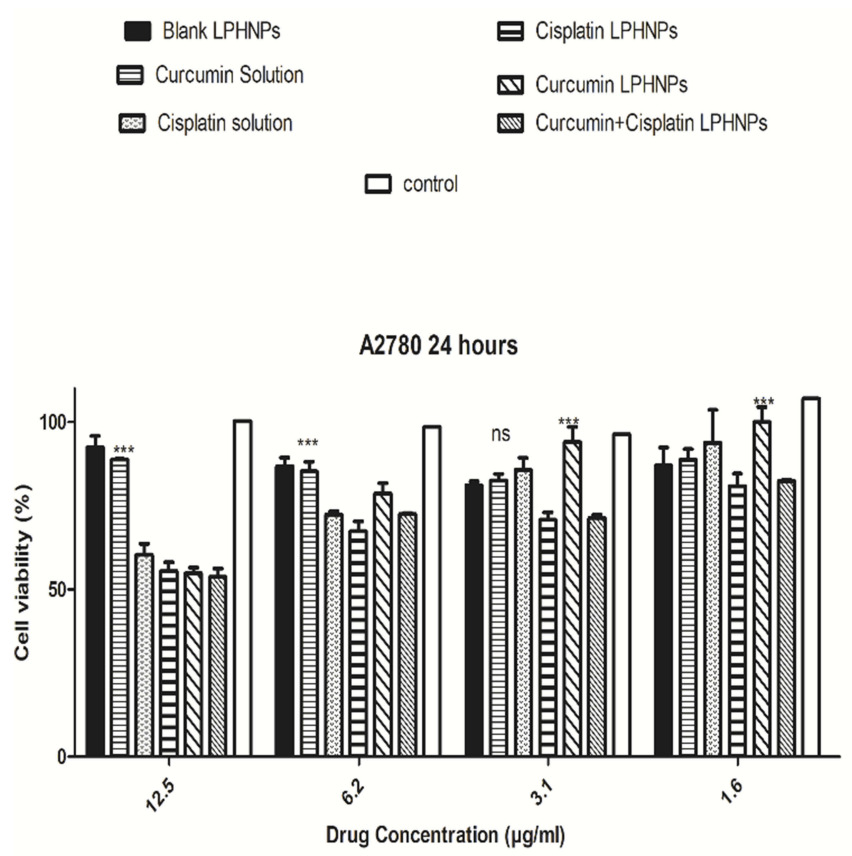

\section{Cell Viability Studies}

Cell viability studies were conducted to evaluate the therapeutic efficacy of curcumin and cisplatin co-loaded hybrid nanoparticles. The cytotoxicity was observed on A2780 cell lines after 24 and 48 hrs of treatment. The results indicated that after $24 \mathrm{hrs}$ of treatment there is not much significant increase in cytotoxicity of coloaded LPHNPs compared to cisplatin loaded LPHNPs but there is significant more cytotoxicity of co-loaded LPHNPs compared to curcumin loaded LPHNPs and curcumin solution. There insignificant difference in cytotoxicity after $24 \mathrm{hrs}$ of treatment was because of the controlled release of cisplatin and curcumin from hybrid nanoparticles while free drugs have easy access to the cells.

After $48 \mathrm{hrs}$ of treatment, cisplatin and curcumin co-loaded LPHNPs have more significant cytotoxicity compared to cisplatin loaded LPHNPs and curcumin loaded LPHNPs particularly at concentrations of 6.2 and $3.1 \mu \mathrm{g} / \mathrm{mL}$. This showed that the addition of curcumin to cisplatin nanoparticles increase the chemosensitization of ovarian cells and resulted in higher cytotoxic effects (Figure 4). The curcumin has cytotoxic effect toward cancer cells and the combination leads to synergistic effect and higher cytotoxicity toward ovarian cell lines. ${ }^{42}$ The synergistic anticancer

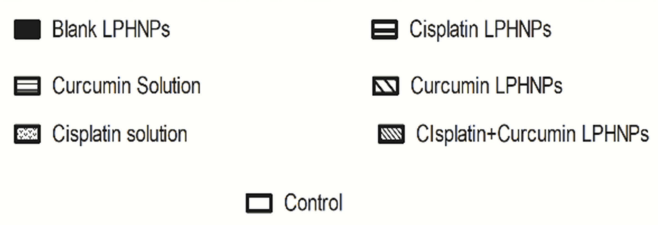

A2780 48 hours

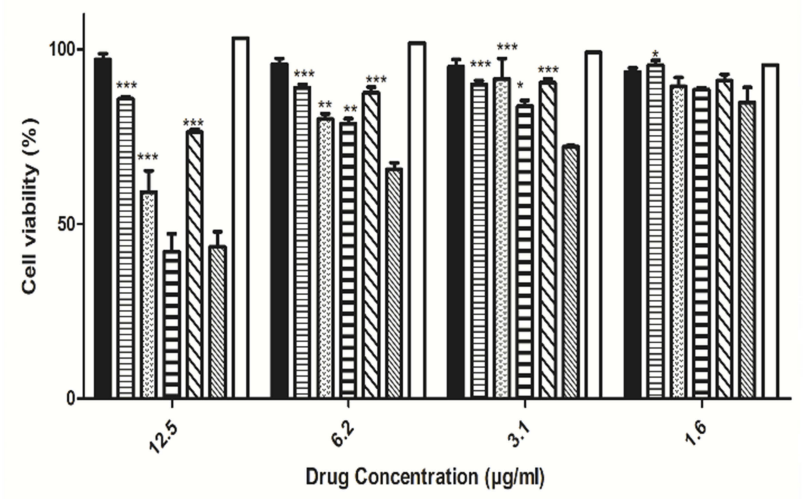

Figure 4 Cytotoxicity studies of cisplatin and curcumin co-loaded LPHNPs on A2780 cell lines after 24 hrs and 48 hrs of treatment. All results indicate mean \pm SD, $n=3$. $* P<0.05$, $* * \mathrm{P}<0.01$, *** $\mathrm{P}<0.001$. 


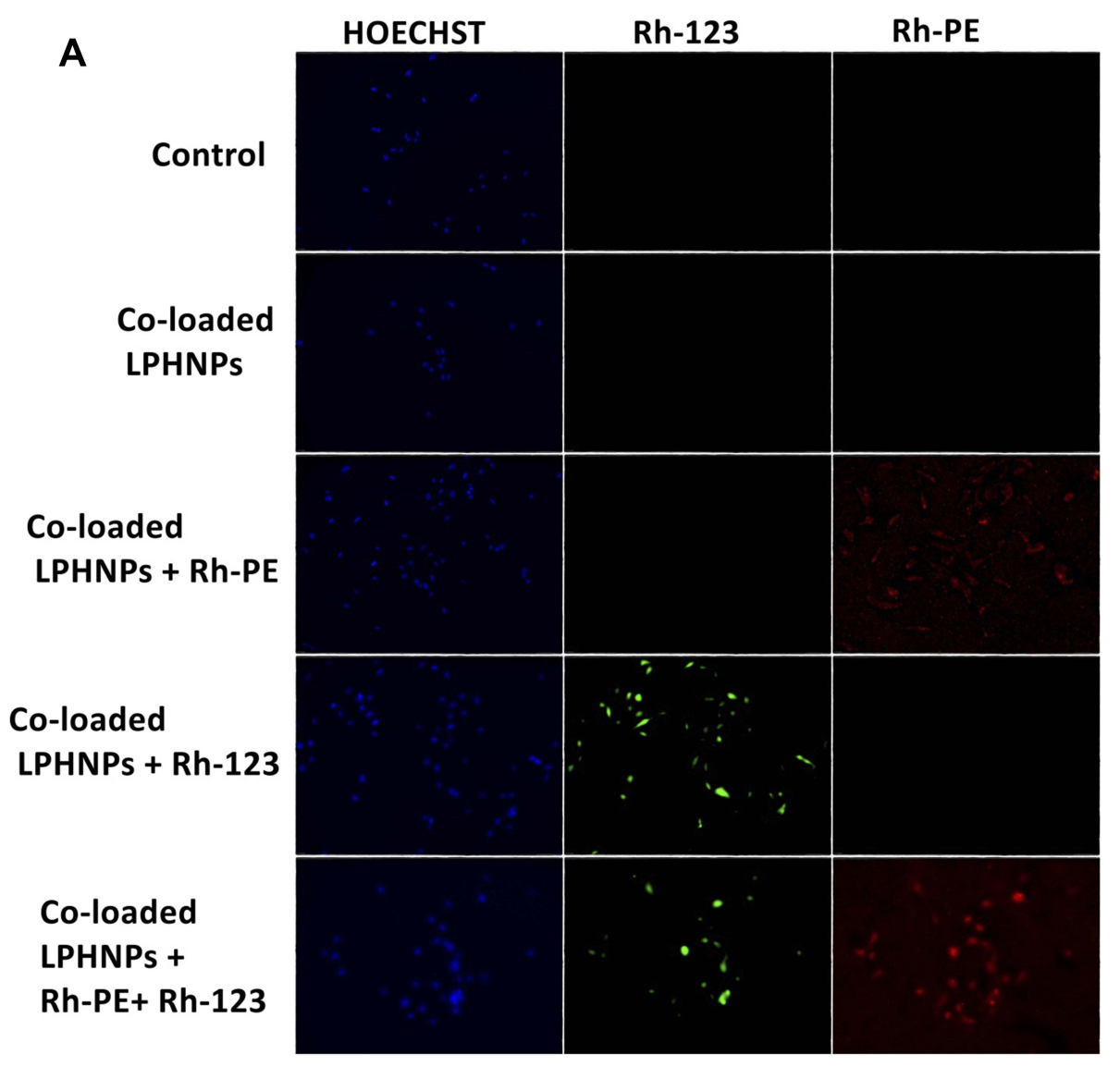

B

Control

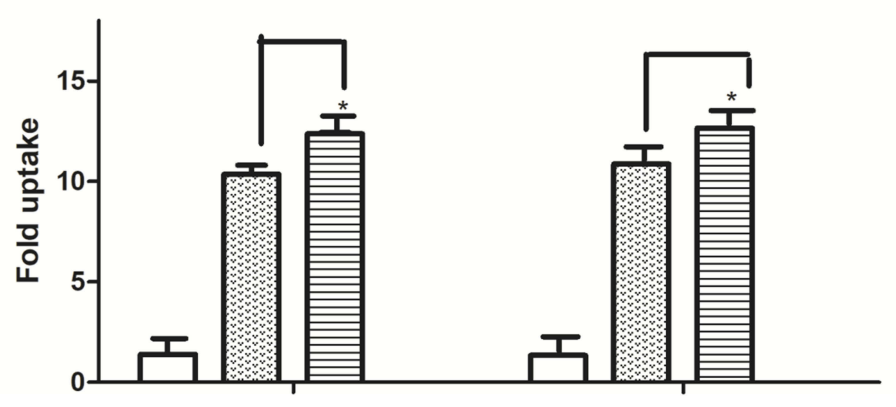

Figure 5 (A) Fluorescence microscopy images of curcumin and cisplatin co-loaded LPHNPs (B) cell uptake of curcumin and cisplatin loaded LPHNPs. All results indicate mean $\pm S D, n=3 . * P<0.05$.

effect of both cisplatin and curcumin also leads to enhanced therapeutic efficacy. ${ }^{43}$ The curcumin arrests the cell cycle at the $\mathrm{G}_{2} / \mathrm{M}$ phase of the cell cycle and cisplatin also inhibit the cells at the $\mathrm{G}_{2} / \mathrm{M}$ phase of the cell cycle. This peculiar combination of cisplatin and curcumin leads to synergistic cytotoxic effects. ${ }^{44}$

\section{Cell Uptake Studies}

The cell uptake studies were conducted to determine the uptake of curcumin and cisplatin co-loaded LPHNPs by A2780 ovarian cell lines.

The uptake of LPHNPs by the cells was determined qualitatively by fluorescence microscopy. The results 

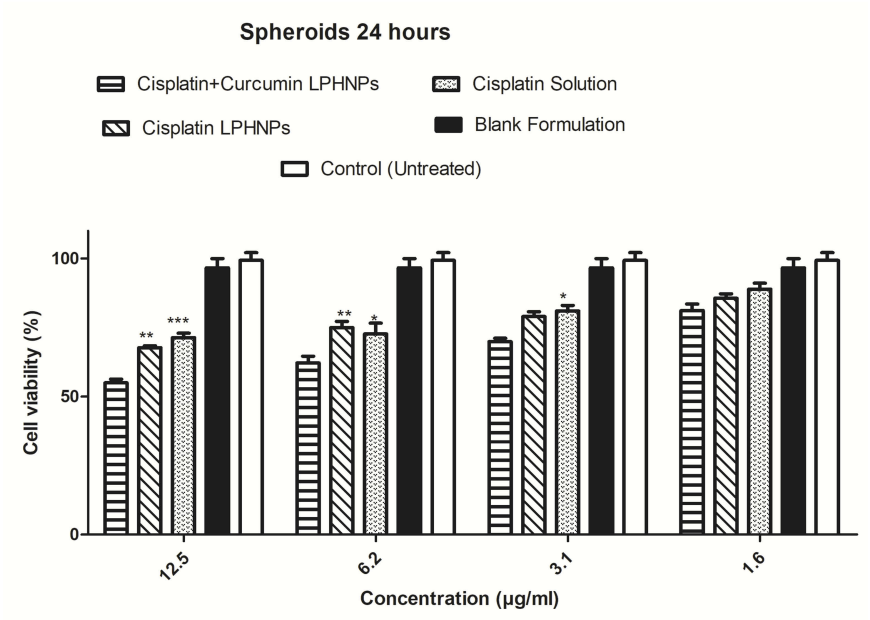

Spheroids 48 hours
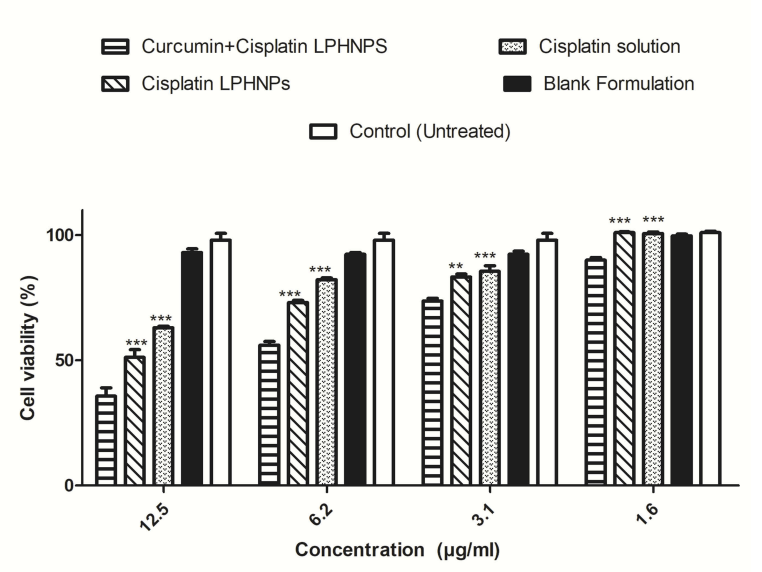

Figure 6 Cytotoxicity studies on 3D spheroids of curcumin and cisplatin co-loaded LPHNPs and cisplatin loaded LPHNPs and cisplatin solution. All results indicate mean \pm $\mathrm{SD}, \mathrm{n}=3$. $* \mathrm{P}<0.05$, ** $\mathrm{P}<0.01$, *** $\mathrm{P}<0.001$.

indicated that co-loaded LPHNPs loaded with fluorescence dye rhodamine-123 produced enhanced green fluorescence, which indicates the uptake of nanoparticles by the cells and hybrid nanoparticles loaded with Rh-PE produces red fluorescence which also confirmed the uptake of nanoparticles by the cells. The addition of curcumin in nanoparticles along with fluorescent dyes produces strong fluorescence (Figure 5A). The curcumin is also known to produce slight green fluorescence hence the cells showed more bright green fluorescence in combination of curcumin and rhodamine-123. The inherent slight green fluorescence of curcumin is also reported in other studies. ${ }^{45}$

To quantitatively determine the uptake of cisplatin loaded LPHNPs and co-loaded LPHNPs and to differentiate the effect of added curcumin on the uptake of nanoparticles, we performed the FACS analysis. The results indicated that there is a 10-fold uptake of cisplatin loaded LPHNPs and 12-fold uptake of curcumin and cisplatin co-loaded LPHNPs by the cells compared to control (Figure 5B). There is a significant increase in uptake as of co-loaded LPHNPs compared to control and cisplatin loaded LPHNPs. These results showed that the addition of curcumin to LPHNPs enhanced their uptake by the cells that lead to higher internalization and increased cytotoxic effect. Previous studies on curcumin nanoparticles also showed enhanced uptake of curcumin loaded nanoparticles by the cells. ${ }^{46}$ The inherent green fluorescence is also detectable quantitatively by FACS analysis and co-loaded LPHNPs showed more uptake than cisplatin loaded LPHNPs with an equal amount (0.5 mole percent) of rhodamine-123 incorporated inside both LPHNPs. ${ }^{47}$

\section{Cytotoxicity Toward 3D Spheroids}

The cytotoxicity studies were conducted on 3D spheroids as a tumor model to evaluate the therapeutic efficacy of co-loaded LPHNPs. The cytotoxicity was observed after one and two days of treatment. The results indicated that blank nanoparticles do not have any cytotoxic effects and are biocompatible. Previous studies showed that 3D spheroids are known to mimic the in-vivo tumor environment and serve as a tool to evaluate the therapeutic efficacy of nanoparticles in the in-vivo environment. ${ }^{48}$

The results indicated that after $24 \mathrm{hrs}$ of treatment co-loaded LPHNPs have more cytotoxic effect compared to cisplatin solution at concentrations of 12.5 , 6.2 and $3.1 \mu \mathrm{g} / \mathrm{mL}$ and is more significant at $12.5 \mu \mathrm{g} /$ $\mathrm{mL}$. Similarly, co-loaded LPHNPs have more cytotoxic effect compared to cisplatin loaded LPHNPs at concentrations of 12.5 and $6.2 \mu \mathrm{g} / \mathrm{mL}$. The co-loaded LPHNPs showed enhanced cytotoxicity even after $24 \mathrm{hrs}$ of incubation compared to monolayer cells because cisplatin solution cannot easily penetrate inside 3D spheroids while co-loaded LPHNPs have been actively taken up by $3 \mathrm{D}$ spheroids.

After $48 \mathrm{hrs}$ of treatment co-loaded LPHNPs produce significant cytotoxic effect compared to cisplatin solution and cisplatin loaded LPHNPs on 3D spheroids. These results indicated that the therapeutic effect of cisplatin in increased by the addition of curcumin, which is known to reduce the resistance of cisplatin (Figure 6). The higher cytotoxicity is observed after $48 \mathrm{hrs}$ of treatment due to the controlled release of curcumin and cisplatin from the 


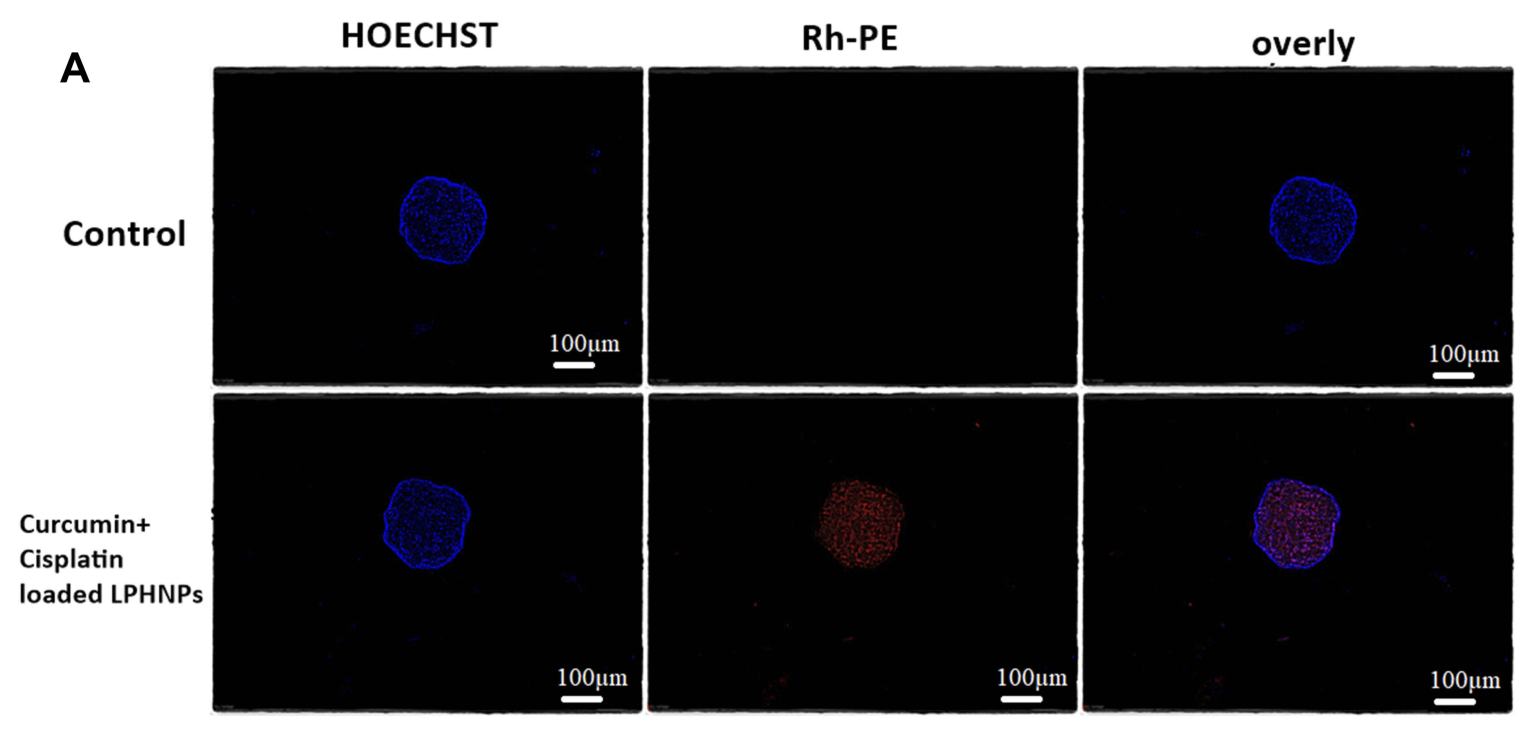

B

Cisplatin LPHNPs+Rh-123 Curcumin+Cisplat LPHNPs+Rh-123

\section{$\square$ Control}

\section{Spheroids}

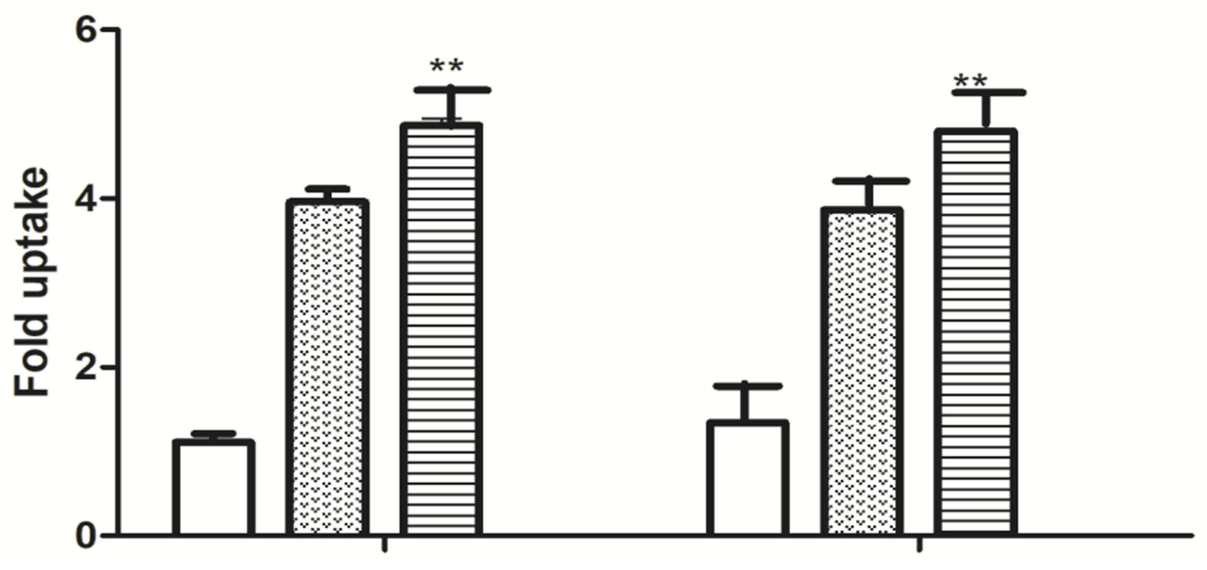

Figure 7 (A) Fluorescence microscopy images of curcumin+ cisplatin co-loaded LPHNPs (B) Cell uptake studies of curcumin + cisplatin co-loaded LPHNPs. All results indicate mean $\pm S D, n=3$. **P $<0.01$.

hybrid nanoparticle. Previous studies on 3D spheroids also showed the enhanced cytotoxic effect of co-loaded curcumin nanoparticles. ${ }^{49}$

\section{Cell Uptake Studies of 3D Spheroids}

The uptake of curcumin and cisplatin loaded LPHNPs by 3D spheroids was evaluated qualitatively using fluorescence microscopy. The fluorescent dye rhodamine-PE was incorporated into the co-loaded LPHNPs and fluorescence microscopy images of spheroids showed that there is significant uptake of co-loaded LPHNPs compared to control (Figure 7A).

To quantitatively determine the uptake of co-loaded LPHNPs and to compare with cisplatin loaded LPHNPs we performed FACS analysis. The results indicated that there is a 4-fold increase in uptake of cisplatin loaded LPHNPs and there is a 5-fold increase in uptake of co-loaded LPHNPs compared to control (Figure 7B). The results indicated that there was a slight increase in uptake by the addition of curcumin. 
The co-loaded LPHNPs have successfully crossed that upper layer of 3D spheroids and penetrated into the deeper layer and produce fluorescence. ${ }^{50}$ The co-loaded LPHNPs showed enhanced cellular internalization due to non-specific interaction with the cell membrane and lead to enhanced fluorescence. ${ }^{51}$

\section{Conclusion}

Lipid-chitosan hybrid nanoparticles were successfully fabricated for co-delivery of cisplatin and curcumin. The nanoparticles with different ratios of lipid and chitosan were prepared with suitable size and enhanced encapsulation efficiency. The encapsulation of curcumin increased with an increase in lipid concentration. The drug release from the LPHNPs was in a controlled manner and the absence of burst release indicated proper encapsulation of both curcumin and cisplatin. The combination of lipid and polymer served as an excellent vehicle for the controlled release of drugs. Polymer provides structural integrity and lipid layer prevents leakage of drug from the LPHNPs. The in vitro cytotoxicity studies revealed enhanced therapeutic efficacy of co-loaded LPHNPs compared to cisplatin loaded LPHNPs and curcumin loaded LPHNPs. The cell uptake studies revealed enhanced uptake of co-loaded LPHNPS. The therapeutic efficacy of co-loaded LPHNPs was further evaluated on 3D spheroids as an in-vivo tumor model. It was concluded that co-loaded LPHNPs showed enhanced cytotoxicity and the addition of curcumin to cisplatin leads to increased chemosensitization of 3D spheroids toward cisplatin.

\section{Acknowledgments}

The authors acknowledge the International Research Support Initiative Program (IRSIP) of the Higher Education Commission of Pakistan for the travel grant to Northeastern University, Boston, USA. The authors also acknowledge support by center of Pharmaceutical Biotechnology and Nanomedicines, Northeastern University, Boston, MA, USA.

\section{Disclosure}

The authors report no conflicts of interest in this work.

\section{References}

1. Yallapu MM, Gupta BK, Jaggi M, Chauhan SC. Fabrication of curcumin encapsulated PLGA nanoparticles for improved therapeutic effects in metastatic cancer cells. J Colloid Interface Sci. 2010;351(1):19-29. doi:10.1016/j.jcis.2010.05.022
2. Wentzensen N, Poole EM, Trabert B, et al. Ovarian cancer risk factors by histologic subtype: an analysis from the ovarian cancer cohort consortium. J Clin Oncol. 2016;34(24):2888. doi:10.1200/JCO.2016.66.8178

3. Agarwal R, Kaye SB. Ovarian cancer: strategies for overcoming resistance to chemotherapy. Nat Rev Cancer. 2003;3(7):502. doi: $10.1038 /$ nrc1123

4. Herzog TJ, Pothuri B. Ovarian cancer: a focus on management of recurrent disease. Nat Rev Clin Oncol. 2006;3(11):604. doi:10.1038/ ncponc0637

5. Nishino H, Satomi Y, Tokuda H, Masuda M. Cancer control by phytochemicals. Curr Pharm Des. 2007;13(33):3394. doi:10.2174/ 138161207782360519

6. Yallapu MM, Jaggi M, Chauhan SC. Curcumin nanoformulations: a future nanomedicine for cancer. Drug Discov Today. 2012;17(12):71-80. doi:10.1016/j.drudis.2011.09.009

7. Kuhar M, Imran S, Singh N. Curcumin and quercetin combined with cisplatin to induce apoptosis in human laryngeal carcinoma Hep-2 cells through the mitochondrial pathway. J Cancer Mol. 2007;3(4):121-128.

8. Aggarwal BB, Bhatt ID, Ichikawa H, et al. 10 Curcumin-Biological and Medicinal Properties; 2006.

9. Goel A, Kunnumakkara AB, Aggarwal BB. Curcumin as "Curecumin": from kitchen to clinic. Biochem Pharmacol. 2008;75(4):787-809. doi:10.1016/j.bcp.2007.08.016

10. Somasundaram S, Edmund NA, Moore DT, Small GW, Shi YY, Orlowski RZ. Dietary curcumin inhibits chemotherapy-induced apoptosis in models of human breast cancer. Cancer Res. 2002;62(13):3868-3875.

11. Nakagawa-Goto K, Yamada K, Nakamura S, et al. Antitumor agents. 258. Syntheses and evaluation of dietary antioxidant - taxoid conjugates as novel cytotoxic agents. Bioorg Med Chem Lett. 2007;17 (18):5204-5209. doi:10.1016/j.bmcl.2007.06.083

12. Guo F, Xu C. Progress on the study of mechanism of the direct action of TCM bioactive components on ovarian cancer. Zhongguo Zhong Xi Yi Jie He Za Zhi. 2005;25(12):1140-1144.

13. Anand P, Sundaram C, Jhurani S, Kunnumakkara AB, Aggarwal BB. Curcumin and cancer: an "old-age" disease with an "age-old" solution. Cancer Lett. 2008;267(1):133-164. doi:10.1016/j.canlet.2008.03.025

14. Sharma R, Gescher A, Steward W. Curcumin: the story so far. Eur $J$ Cancer. 2005;41(13):1955-1968. doi:10.1016/j.ejca.2005.05.009

15. Cheng L, Jin C, Lv W, Ding Q, Han X. Developing a highly stable PLGAmPEG nanoparticle loaded with cisplatin for chemotherapy of ovarian cancer. PLoS One. 2011;6(9):e25433. doi:10.1371/journal.pone.0025433

16. Yallapu MM, Maher DM, Sundram V, Bell MC, Jaggi M, Chauhan SC. Curcumin induces chemo/radio-sensitization in ovarian cancer cells and curcumin nanoparticles inhibit ovarian cancer cell growth. $J$ Ovarian Res. 2010;3(1):11. doi:10.1186/1757-2215-3-11

17. Kuhad A, Pilkhwal S, Sharma S, Tirkey N, Chopra K. Effect of curcumin on inflammation and oxidative stress in cisplatin-induced experimental nephrotoxicity. J Agric Food Chem. 2007;55 (25):10150-10155. doi:10.1021/jf0723965

18. Youm I, West MB, Li W, Du X, Ewert DL, Kopke RD. siRNA-loaded biodegradable nanocarriers for therapeutic MAPK1 silencing against cisplatin-induced ototoxicity. Int $J$ Pharm. 2017;528(1-2):611-623. doi:10.1016/j.ijpharm.2017.06.035

19. Zhang J, Liu J, Xu X, Li L. Curcumin suppresses cisplatin resistance development partly via modulating extracellular vesicle-mediated transfer of MEG3 and miR-214 in ovarian cancer. Cancer Chemother Pharmacol. 2017;79(3):479-487. doi:10.1007/s00280-017-3238-4

20. Sinha R, Kim GJ, Nie S, Shin DM. Nanotechnology in cancer therapeutics: bioconjugated nanoparticles for drug delivery. Mol Cancer Ther. 2006;5(8):1909-1917. doi:10.1158/1535-7163.MCT-06-0141

21. Misra R, Acharya S, Sahoo SK. Cancer nanotechnology: application of nanotechnology in cancer therapy. Drug Discov Today. 2010;15 (19-20):842-850. doi:10.1016/j.drudis.2010.08.006

22. Mandal B, Bhattacharjee H, Mittal N, et al. Core-shell-type lipidpolymer hybrid nanoparticles as a drug delivery platform. Nanomed. 2013;9(4):474-491. doi:10.1016/j.nano.2012.11.010 
23. Zhang L, Chan JM, Gu FX, et al. Self-assembled lipid- polymer hybrid nanoparticles: a robust drug delivery platform. ACS Nano. 2008;2(8):1696-1702. doi:10.1021/nn800275r

24. De Miguel I, Imbertie L, Rieumajou V, Major M, Kravtzoff R, Betbeder D. Proofs of the structure of lipid coated nanoparticles (SMBVTM) used as drug carriers. Pharm Res. 2000;17(7):817-824. doi:10.1023/A:1007504124603

25. Hu Q, Bae M, Fleming E, Lee J-Y LY. Biocompatible polymeric nanoparticles with exceptional gastrointestinal stability as oral delivery vehicles for lipophilic bioactives. Food Hydrocoll. 2019;89:386395. doi:10.1016/j.foodhyd.2018.10.057

26. Bhise KS, Dhumal RS, Paradkar AR, Kadam SS. Effect of drying methods on swelling, erosion and drug release from chitosannaproxen sodium complexes. AAPS PharmSciTech. 2008;9(1):1-12. doi:10.1208/s12249-007-9001-0

27. Sun W, Mao S, Wang Y, et al. Bioadhesion and oral absorption of enoxaparin nanocomplexes. Int J Pharm. 2010;386(1-2):275-281. doi:10.1016/j.ijpharm.2009.11.025

28. Elgadir MA, Uddin MS, Ferdosh S, Adam A, Chowdhury AJK, Sarker MZI. Impact of chitosan composites and chitosan nanoparticle composites on various drug delivery systems: a review. J Food Drug Anal. 2015;23(4):619-629. doi:10.1016/j.jfda.2014.10.008

29. Sonvico F, Cagnani A, Rossi A, et al. Formation of self-organized nanoparticles by lecithin/chitosan ionic interaction. Int J Pharm. 2006;324(1):67-73. doi:10.1016/j.ijpharm.2006.06.036

30. Xu P, Van Kirk EA, Li S, et al. Highly stable core-surface-crosslinked nanoparticles as cisplatin carriers for cancer chemotherapy. Colloids Surf B Biointerfaces. 2006;48(1):50-57. doi:10.1016/j.colsurfb.2006.01.004

31. Gabano E, Ravera M, Zanellato I, et al. An unsymmetric cisplatinbased Pt (IV) derivative containing 2-(2-propynyl) octanoate: a very efficient multi-action antitumor prodrug candidate. Dalton Trans. 2017;46(41):14174-14185. doi:10.1039/C7DT02928D

32. Garcea G, Jones D, Singh R, et al. Detection of curcumin and its metabolites in hepatic tissue and portal blood of patients following oral administration. Br J Cancer. 2004;90(5):1011. doi:10.1038/sj.bjc.6601623

33. Pan J, Mendes LP, Yao M, et al. Polyamidoamine dendrimers-based nanomedicine for combination therapy with siRNA and chemotherapeutics to overcome multidrug resistance. Eur J Pharm Biopharm. 2019;136:18-28. doi:10.1016/j.ejpb.2019.01.006

34. Oliveira MS, Aryasomayajula B, Pattni B, Mussi SV, Ferreira LA, Torchilin VP. Solid lipid nanoparticles co-loaded with doxorubicin and $\alpha$-tocopherol succinate are effective against drug-resistant cancer cells in monolayer and 3-D spheroid cancer cell models. Int J Pharm. 2016;512(1):292-300. doi:10.1016/j.ijpharm.2016.08.049

35. Henriksen I, Våagen SR, Sande SA, Smistad G, Karlsen J. Interactions between liposomes and chitosan II: effect of selected parameters on aggregation and leakage. Int J Pharm. 1997;146 (2):193-203. doi:10.1016/S0378-5173(96)04801-6

36. Grant J, Blicker M, Piquette-Miller M, Allen C. Hybrid films from blends of chitosan and egg phosphatidylcholine for localized delivery of paclitaxel. J Pharm Sci. 2005;94(7):1512-1527. doi:10.1002/jps.20379

37. Duan J, Zhang Y, Han S, et al. Synthesis and in vitro/in vivo anticancer evaluation of curcumin-loaded chitosan/poly (butyl cyanoacrylate) nanoparticles. Int $J$ Pharm. 2010;400(1-2):211-220. doi:10.1016/j.ijpharm.2010.08.033
38. Zhang J, Li J, Shi Z, et al. pH-sensitive polymeric nanoparticles for co-delivery of doxorubicin and curcumin to treat cancer via enhanced pro-apoptotic and anti-angiogenic activities. Acta Biomater. 2017;58:349-364. doi:10.1016/j.actbio.2017.04.029

39. Das RK, Kasoju N, Bora U. Encapsulation of curcumin in alginatechitosan-pluronic composite nanoparticles for delivery to cancer cells. Nanomed. 2010;6(1):153-160. doi:10.1016/j.nano.2009.05.009

40. Anitha A, Maya S, Deepa N, Chennazhi K, Nair S, Jayakumar R. Curcumin-loaded N, O-carboxymethyl chitosan nanoparticles for cancer drug delivery. J Biomater Sci Polym Ed. 2012;23(11):1381-1400.

41. Chan JM, Zhang L, Yuet KP, et al. PLGA-lecithin-PEG core-shell nanoparticles for controlled drug delivery. Biomaterials. 2009;30 (8):1627-1634. doi:10.1016/j.biomaterials.2008.12.013

42. Cheng Y, Zhao P, Wu S, et al. Cisplatin and curcumin co-loaded nano-liposomes for the treatment of hepatocellular carcinoma. Int J Pharm. 2018;545(1-2):261-273. doi:10.1016/j.ijpharm.2018.05. 007

43. Baharuddin P, Satar N, Fakiruddin KS, et al. Curcumin improves the efficacy of cisplatin by targeting cancer stem-like cells through p21 and cyclin D1-mediated tumour cell inhibition in non-small cell lung cancer cell lines. Oncol Rep. 2016;35(1):13-25. doi:10.3892/or.2015.4371

44. Mori H, Niwa K, Zheng Q, Yamada Y, Sakata K, Yoshimi N. Cell proliferation in cancer prevention; effects of preventive agents on estrogen-related endometrial carcinogenesis model and on an in vitro model in human colorectal cells. Mutat Res. 2001;480:201-207. doi:10.1016/S0027-5107(01)00200-7

45. Xu Y-Q, Chen W-R, Tsosie JK, et al. Niosome encapsulation of curcumin: characterization and cytotoxic effect on ovarian cancer cells. J Nanomater. 2016;2016.

46. Saxena V, Hussain MD. Polymeric mixed micelles for delivery of curcumin to multidrug resistant ovarian cancer. $J$ Biomed Nanotechnol. 2013;9(7):1146-1154. doi:10.1166/jbn.2013.1632

47. Yallapu MM, Jaggi M, Chauhan SC. $\beta$-Cyclodextrin-curcumin selfassembly enhances curcumin delivery in prostate cancer cells. Colloids Surf B Biointerfaces. 2010;79(1):113-125. doi:10.1016/j. colsurfb.2010.03.039

48. Patel NR, Aryasomayajula B, Abouzeid AH, Torchilin VP. Cancer cell spheroids for screening of chemotherapeutics and drug-delivery systems. Ther Deliv. 2015;6(4):509-520. doi:10.4155/tde.15.1

49. Kumari P, Rompicharla SVK, Muddineti OS, Ghosh B, Biswas S Transferrin-anchored poly (lactide) based micelles to improve anticancer activity of curcumin in hepatic and cervical cancer cell monolayers and 3D spheroids. Int J Biol Macromol. 2018;116:1196-1213. doi:10.1016/j.ijbiomac.2018.05.040

50. Sarisozen C, Dhokai S, Tsikudo EG, Luther E, Rachman IM, Torchilin VP. Nanomedicine based curcumin and doxorubicin combination treatment of glioblastoma with scFv-targeted micelles: in vitro evaluation on 2D and 3D tumor models. Eur J Pharm Biopharm. 2016;108:54-67. doi:10.1016/j.ejpb.2016.08.013

51. Muddineti OS, Kumari P, Ray E, Ghosh B, Biswas S. Curcuminloaded chitosan-cholesterol micelles: evaluation in monolayers and 3D cancer spheroid model. Nanomedicine. 2017;12(12):1435-1453. doi:10.2217/nnm-2017-0036

International Journal of Nanomedicine

Dovepress

\section{Publish your work in this journal}

The International Journal of Nanomedicine is an international, peerreviewed journal focusing on the application of nanotechnology in diagnostics, therapeutics, and drug delivery systems throughout the biomedical field. This journal is indexed on PubMed Central, MedLine, CAS, SciSearch ${ }^{\mathbb{R}}$, Current Contents ${ }^{\mathbb{B}} /$ Clinical Medicine,

Journal Citation Reports/Science Edition, EMBase, Scopus and the Elsevier Bibliographic databases. The manuscript management system is completely online and includes a very quick and fair peer-review system, which is all easy to use. Visit http://www.dovepress.com/ testimonials.php to read real quotes from published authors. 